\title{
Efficacy and Safety of Abdominal Trunk Muscle Strengthening Using an Innovative Device in Elderly Patients With Chronic Low Back Pain: A Pilot Study
}

\author{
Satoshi Kato, MD, Satoru Demura, MD, Yuki Kurokawa, MD, PhD, Naoki Takahashi, MD, \\ Kazuya Shinmura, MD, Noriaki Yokogawa, MD, Noritaka Yonezawa, MD, Takaki Shimizu, MD, \\ Ryo Kitagawa, MD, Hiroyuki Tsuchiya, MD
}

Department of Orthopaedic Surgery, Graduate School of Medical Sciences, Kanazawa University, Kanazawa, Japan

Objective To examine the efficacy and safety of an innovative, device-driven abdominal trunk muscle strengthening program, with the ability to measure muscle strength, to treat chronic low back pain (LBP) in elderly participants. Methods Seven women with non-specific chronic LBP, lasting at least 3 months, were enrolled and treated with the prescribed exercise regimen. Patients participated in a 12-week device-driven exercise program which included abdominal trunk muscle strengthening and 4 types of stretches for the trunk and lower extremities. Primary outcomes were adverse events associated with the exercise program, improvement in abdominal trunk muscle strength, as measured by the device, and improvement in the numerical rating scale (NRS) scores of LBP with the exercise. Secondary outcomes were improvement in the Roland-Morris Disability Questionnaire (RDQ) score and the results of the locomotive syndrome risk test, including the stand-up and two-step tests.

Results There were no reports of increased back pain or new-onset abdominal pain or discomfort during or after the device-driven exercise program. The mean abdominal trunk muscle strength, NRS, RDQ scores, and the stand-up and two-step test scores were significantly improved at the end of the trial compared to baseline.

Conclusion No participants experienced adverse events during the 12-week strengthening program, which involved the use of our device and stretching, indicating the program was safe. Further, the program significantly improved various measures of LBP and physical function in elderly participants.

Keywords Abdominal muscles, Elderly, Low back pain, Physical therapy, Strengthening

Received July 29, 2019; Revised September 19, 2019; Accepted November 11, 2019; Published online May 29, 2020

Corresponding author: Satoshi Kato

Department of Orthopaedic Surgery, Graduate School of Medical Sciences, Kanazawa University, 13-1 Takara-machi, Kanazawa 920-8641, Japan. Tel: +81-76-265-2374, Fax: +81-76-234-4261, E-mail: skato323@gmail.com

ORCID: Satoshi Kato (https://orcid.org/0000-0003-4762-5932); Satoru Demura (https://orcid.org/0000-0002-5703-3802); Yuki Kurokawa (https:// orcid.org/0000-0001-5665-4829); Naoki Takahashi (https://orcid.org/0000-0002-0177-8573); Kazuya Shinmura (https://orcid.org/0000-0002-85834615); Noriaki Yokogawa (https://orcid.org/0000-0002-3415-5823); Noritaka Yonezawa (https://orcid.org/0000-0002-8037-6213); Takaki Shimizu (https://orcid.org/0000-0001-7681-0593); Ryo Kitagawa (https://orcid.org/0000-0002-3947-3193); Hiroyuki Tsuchiya (https://orcid.org/0000-00030730-7921)

(a) This is an open-access article distributed under the terms of the Creative Commons Attribution Non-Commercial License (http://creativecommons.org/ licenses/by-nc/4.0) which permits unrestricted noncommercial use, distribution, and reproduction in any medium, provided the original work is properly cited. Copyright ( 2020 by Korean Academy of Rehabilitation Medicine 


\section{INTRODUCTION}

The economic and social burdens of low back pain (LBP) are considerable, and growing [1]. Among elderly adults, LBP is the most frequently reported musculoskeletal complaint and the third most frequently reported symptom of any kind $[2,3]$. Although multiple clinical interventions are available to treat chronic low back pain (CLBP), only a few have been proven effective [4-7]. Exercise is clearly effective for treating CLBP [6-11]. A systematic review by Hayden et al. [11] reported that strengthening exercises are the most effective options for improving functional outcomes among the various types of exercise therapies. Unfortunately, motivation and adherence to exercise therapies are generally low among elderly patients with CLBP [12]. Exercise requires a much longer time to decrease pain than oral medications or injections, which are often prescribed to elderly patients. Elderly patients with CLBP often report they cannot, or will not, exercise owing to mobility difficulties associated with loss of strength, flexibility, or endurance, and presence of pain and/or deformities in the spine and extremities $[13,14]$. These problems reduce exercise adherence in elderly patients with CLBP, thus diminishing the potential effects. Hence, important considerations for CLBP exercise therapy include the ability of the patient to perform the exercise easily and repeatedly to achieve early and recognizable effects while meeting short-term goals [12].

Locomotive syndrome is characterized by restricted or limited ability to walk due to degenerative dysfunction of locomotive organs [15]. Syndrome progression can impair activities of daily living and increase nursing care needs. Exercise interventions for locomotive syndrome are effective for improving physical function. However, we should be careful when choosing the type and intensity of exercise because most patients are elderly and also exhibit degenerative musculoskeletal system dysfunction [16].

We developed a novel exercise device for the abdominal trunk muscles (Nippon Sigmax Co. Ltd., Tokyo, Japan) (Fig. 1). This device allows patients to perform abdominal trunk muscle strengthening exercises while in a sitting position, and requires no trunk movement. The device also enables patients to measure their abdominal trunk muscle strength. Additionally, exercises and strength measures carried out with the device do not induce stress and/or pain in the lumbar spine or the extremities. A prior validation study demonstrated that the device could measure muscle strength and featured excellent intraand inter-rater reliability $[17,18]$. Another study found that strength training with the device increased both the strength and activation of the abdominal trunk muscles, including the diaphragm, abdominal rectus, external and internal obliques, transverse abdominal muscles, and pelvic floor muscles [18]. However, the authors did not evaluate the efficacy of exercise that used the device for treatment of CLBP treatment. Determining the efficacy and safety of device use by elderly patients is particularly important in terms of clinical utility.

The purpose of this pilot study was to examine the efficacy and safety of exercise using the device for treatment of CLBP in elderly patients.

\section{MATERIALS AND METHODS}

\section{Ethics statement}

Our university hospital ethics committee approved this trial (No. 2016-009). Written informed consent was obtained from each prospective participant before registration by research physicians, in accordance with the Declaration of Helsinki (Clinical trial registration: UMIN000023181).
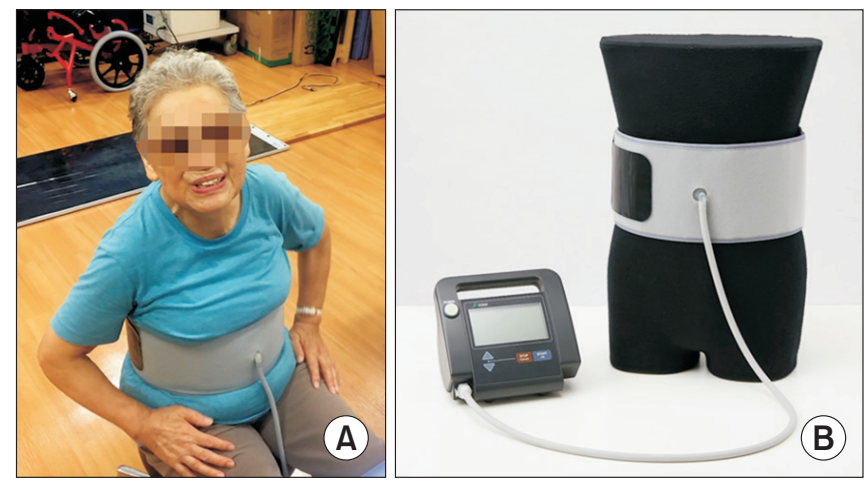

Fig. 1. Innovative exercise device for the abdominal trunk muscle. (A) Photograph of a device-equipped patient. Using the device, the patient can measure their abdominal trunk muscle strength or perform strengthening exercise in sitting position without requiring trunk movement. (B) Photograph of the device. It consists of an inflatable cuff and a mechanical manometer to measure pressure. 


\section{Participants}

This trial was conducted between July 2016 and July 2017 in the Department of Orthopaedic Surgery and Rehabilitation within our university hospital. All participants were diagnosed with CLBP by a physician and referred to our institute. Inclusion criteria were diagnosis of CLBP, of at least 3 months duration, by a physician; age $\geq 65$ years; moderate or severe CLBP (LBP intensity $\geq 3$ based on an 11-point numerical rating scale [NRS] pain score, no pain $=0$, and worst pain $=10$ ) at study registration; capable of performing the prescribed exercise regimen in this trial, and capable of understanding the content of the trial and providing informed consent after having the trial explained by a physician. We excluded individuals with

Table 1. Participant characteristics $(n=7)$

\begin{tabular}{lc}
\hline \multicolumn{1}{c}{ Characteristic } & Value \\
\hline Age $(\mathrm{yr})$ & $75.4 \pm 6.1(68-84)$ \\
Height $(\mathrm{cm})$ & $151.0 \pm 7.4(139-161)$ \\
Weight $(\mathrm{kg})$ & $56.9 \pm 8.9(45-72)$ \\
BMI $\left(\mathrm{kg} / \mathrm{cm}^{2}\right)$ & $24.9 \pm 3.1(22.0-31.6)$ \\
NRS of CLBP at the start of the trial & $5.4 \pm 1.6(4-9)$ \\
\hline
\end{tabular}

Values are presented as mean \pm standard deviation (range). BMI, body mass index; NRS, 11-point numerical rating scale; CLBP, chronic low back pain.

Table 2. Inclusion/exclusion criteria of the study

\begin{tabular}{l}
\hline Inclusion criteria \\
- Diagnosis of CLBP lasting at least 3 months by a \\
physician \\
.65-year-old or older \\
- Moderate or severe CLBP: 3 or more by NRS pain \\
score at study registration \\
- Capable of performing the prescribed exercise regi- \\
men in this trial \\
- Capable of understanding the content of the trial, \\
and giving the informed consent \\
Exclusion criteria \\
- Significant neurological sign or specific spinal pa- \\
thology \\
- History of spinal surgery \\
. Severe osteoporotic spine \\
. Comorbidity of severe medical diseases, rheumato- \\
logical disease, or dementia
\end{tabular}

CLBP, chronic low back pain; NRS, 11-point numerical rating scale. significant neurological signs or specific spinal pathologies (e.g., malignancy, infection, acute vertebral fracture), history of spinal surgery, severe osteoporotic spine, severe medical comorbidities (e.g., cardiovascular, respiratory, or renal disease), comorbid rheumatologic disease, or comorbid dementia (Tables 1,2).

\section{Exercise device}

The device resembles a sphygmomanometer, with an inflatable cuff and a mechanical manometer for measuring pressure [17] (Fig. 1). To take a measurement while seated, the cuff is placed around the participant's abdomen, inflated, and pressure (i.e., the baseline pressure; Fig. 2) is applied to the abdominal wall. An electrically operated pump inflates the cuff until adequate resistance from the abdominal wall is detected. The magnitude of the baseline pressure is set based on the participant's preference. Under this baseline pressure, the participant generates his or her maximum force by contracting the abdominal trunk muscles. The cuff pressure increases and eventually peaks (i.e., the peak pressure; Fig. 2). At this point, the manometer calculates and reports a pressure value. This value is obtained by subtracting the baseline pressure from the peak pressure, thereby producing an estimate of abdominal trunk muscle strength. After the pressure peaks, it decreases automatically while

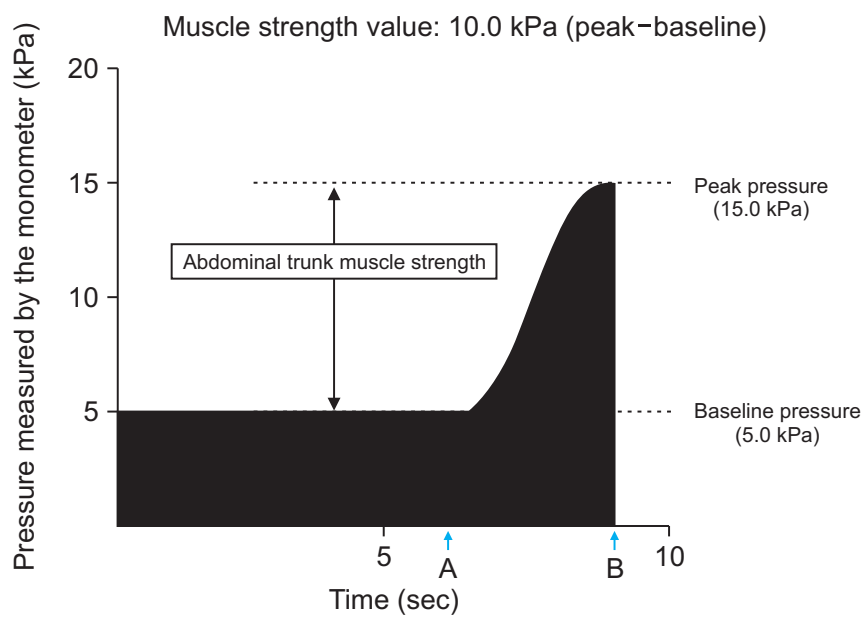

Fig. 2. Time course of the pressure value calculated by the mechanical manometer of the device during the measurement of abdominal trunk muscle strength. " $\mathrm{A}$ " indicates the time point when the patient's abdominal muscles begin to contract against the pressure. "B" shows the reduction in pressure in the cuff after the peak pressure is attained. 
the air in the cuff is released. The muscle strength value was considered as an estimate of the abdominal trunk muscle strength.

While using the device for muscle strengthening, the

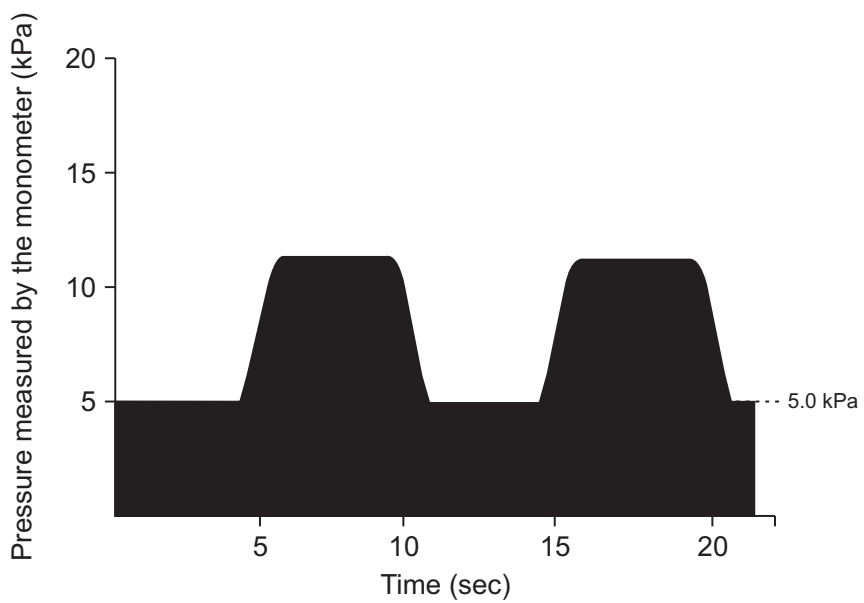

Fig. 3. Time course of the pressure value calculated by the mechanical manometer of the device during abdominal trunk muscle strengthening. The participants exerted the force necessary for the pressure in the cuff to reach $50 \%$ to $80 \%$ of the peak pressure measured at the beginning of the exercise. Intermittent muscle contractions were performed once every 10 seconds, with 5 seconds of muscle contraction and 5 seconds of rest in the 10-minute session. participant contracts his or her abdominal trunk muscles intermittently, or continually, in opposition to cuff pressure. This exercise resembles a bracing exercise and is stabilizing [19]. When the cuff pressure peaks, the participant performs isometric and maximum muscle contractions under maximum pressure from the cuff. During the strengthening exercise and during muscle strength measurement, isometric or eccentric abdominal trunk muscle contraction occurs under the cuff pressure. These device-driven exercises allow participants to contract their abdominal trunk muscles easily and with increased power.

\section{Run-in period (preparation)}

A run-in period of 2 weeks was designed to ensure the stability of the participants with CLBP before starting the intervention. During the 2-week run-in period and the 12-week trial period, all participants were asked to discontinue any pain medications-except loxoprofen sodium, other exercises, and local injections-if they were receiving these treatments. As a result, none of the 7 participants took any pain relievers during the run-in or trial period. However, loxoprofen sodium was allowed during the run-in and study periods.
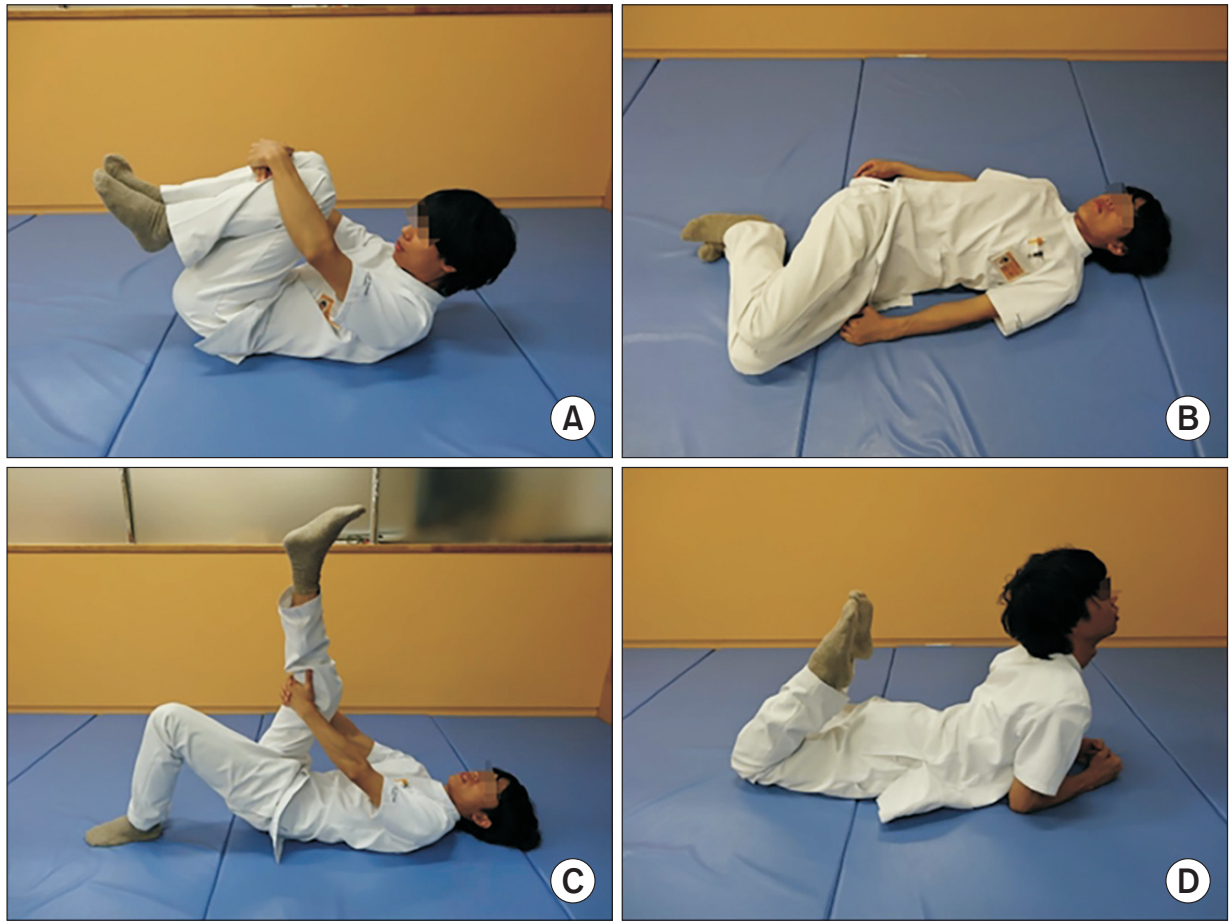

Fig. 4. Four types of stretching exercises conducted in the trial. (A) Lumbar flexion, and knee and hip flexions in the supine position, stretching the back and gluteal muscles. (B) Lumbar rotation in the supine position, stretching the abdominal muscles. (C) Straight leg raising in the supine position, stretching the hamstrings. (D) Lumbar extension and knee flexion in the prone position, stretching the abdominal, iliopsoas, and knee extensor muscles. 


\section{Interventions}

After the run-in period, all participants were transitioned to a 12-week, device-driven exercise program which consisted of strengthening and stretching exercises. The strengthening exercises consisted of abdominal trunk muscle strengthening for 10 minutes per session. Strength measurements using the device were routinely performed before exercise sessions. During the strengthening exercise, each participant was instructed to intermittently contract the abdominal trunk muscles under the cuff pressure and exert the force necessary for the cuff pressure to reach $50 \%-80 \%$ of the peak pressure, as measured at the beginning of the exercise (Fig. 3). Intermittent muscle contractions were performed once every 10 seconds, with 5 seconds of muscle contraction and 5 seconds of rest. The stretching exercise consisted of 4 types of abdominal and back muscle, iliopsoas, gluteal muscle, and hamstring stretches (Fig. 4) along with lumbar spine mobilization. Each strengthening exercise and stretch were performed twice in our hospital's rehabilitation room. Vital signs, including blood pressure and heart rate, were measured prior to, and at the end of, two strengthening sessions. The whole program, including exercises and vital sign measurement, took approximately 30-40 minutes. Participants were asked to visit our hospital three times per week for 12 weeks. Therefore, the program consisted of a total of 36 exercise sessions.

\section{Outcomes}

\section{Primary outcome}

The primary outcomes were adverse events associated with the exercise program, improvements in abdominal trunk muscle strength as measured by the device, and LBP intensity (NRS pain score) during the exercise. Adverse events and muscle strength were evaluated at every visit to our office while the participant performed the exercises. Abnormal vital sign changes were defined as a $>30 \%$ increase in systolic blood pressure and/or heart rate after the strengthening exercise. Patients were asked to rate their LBP using the NRS score over the last 3-5 days. NRS of LBP was evaluated at 0 (start of the intervention), 4, 8, and 12 (end of the intervention) weeks.

\section{Secondary outcome}

Disability and quality of life (QOL) impairment due to LBP were evaluated using the Roland-Morris Disability
Table 3. Scoring system of stand-up test

\begin{tabular}{|lc|}
\hline \multicolumn{1}{|c|}{ Height $\mathbf{( c m )}$} & Score \\
\hline Two-leg stand & \\
\hline Fail at 40 & 0 \\
\hline 40 & 1 \\
30 & 2 \\
20 & 3 \\
\hline 10 & 4 \\
\hline One-leg stand & \\
\hline 40 & 5 \\
\hline 30 & 6 \\
\hline 20 & 7 \\
\hline 10 & 8 \\
\hline
\end{tabular}

One-leg stand requires participants to succeed at indicated height in both right and left leg.

Questionnaire (RDQ). Locomotive syndrome was assessed using the locomotive syndrome risk test comprising the stand-up test, two-step test, and the 25-Question Geriatric Locomotive Function Scale (GLFS-25) [16]. These secondary outcomes were evaluated at 0 and 12 weeks (start and end of the intervention). To evaluate the result of the stand-up test, participants were assigned a score that ranged from 0-8, as shown in Table 3 [20].

\section{Comparisons between good and limited responders}

Based on the improvement in abdominal trunk muscle strength, as measured with the device during the trial, participants were identified as good (those whose muscle strength increased by $\geq 3.0 \mathrm{kPa}$ after the exercise program) or limited (those whose strength increased by $<3.0$ $\mathrm{kPa}$ after the exercise program) responders. Outcomes were compared between the two groups.

\section{Statistical analysis}

Data are presented as means and standard deviations. To evaluate the effects of the intervention, we used the Friedman test to evaluate differences in the primary outcome variables of the abdominal trunk muscle strength trials, as measured by the device and NRS of LBP at $0,4,8$, and 12 weeks. The Wilcoxon signed-ranked test was used to evaluate differences in the secondary outcomes at the beginning ( 0 week) and end ( 12 weeks) of the intervention. The Mann-Whitney U-test was used to evaluate improvements in outcomes between good and limited 
responders. All significance levels were set at 0.05 . SPSS version 19.0 for Windows (IBM Corp., Armonk, NY, USA) was used for statistical analyses.

\section{RESULTS}

Seven participants were enrolled and treated with the prescribed exercise regimen in this trial. All participants were females, with a mean age of 75.4 years (range, 68-84 years). Participants' characteristics and inclusion/exclusion criteria are summarized in Tables 1 and 2 .

\section{Primary outcomes}

None of the 7 participants experienced adverse events during the trial period. There were no reports of increased back pain or new-onset abdominal pain or discomfort during or after the device-driven exercise program. No abnormal changes in blood pressure or heart rate were observed during the exercise program.
The mean abdominal trunk muscle strength was significantly increased at 8 and 12 weeks (end of the trial) compared to 0 week (baseline) and 4 weeks (Table 4). The mean NRS scores of LBP were significantly decreased at 4,8 , and 12 weeks compared to 0 week. The mean NRS score gradually decreased over time, and was lower at 8 weeks than at 4 weeks (Table 4). Based on muscle strength improvement, 4 participants were identified as good responders, and 3 as limited responders (Table 4 ).

\section{Secondary outcomes}

The mean RDQ score was significantly lower at the end of the trial than at the start $(3.9 \pm 3.4$ vs. $6.0 \pm 4.1, \mathrm{p}<0.05)$ (Table 5). With regard to the locomotive syndrome risk test, the mean scores of the stand-up and two-step tests were significantly improved at the end of the trial compared to baseline (Table 5). However, the mean scores of the GLFS-25 at the end of the trial were not significantly increased over baseline.

Table 4. Primary outcomes during the time courses in the trial

\begin{tabular}{|c|c|c|c|c|}
\hline & O week & 4 weeks & 8 weeks & 12 weeks \\
\hline \multicolumn{5}{|l|}{ ATMS (kPa) } \\
\hline All participants $(\mathrm{n}=7)$ & $4.4 \pm 2.1(1.6-6.4)$ & $4.6 \pm 2.1(1.1-6.8)$ & $6.7 \pm 2.9(2.5-9.9)^{\mathrm{a}, \mathrm{b}}$ & $7.8 \pm 3.7(2.5-13.3)^{\mathrm{a}, \mathrm{b}}$ \\
\hline Good responders $(n=4)$ & $4.4 \pm 2.2(1.6-6.3)$ & $5.5 \pm 1.8(2.8-6.8)$ & $7.8 \pm 3.0(3.4-9.9)$ & $9.5 \pm 3.5(5.9-13.3)$ \\
\hline Limited responders $(\mathrm{n}=3)$ & $4.4 \pm 2.4(1.7-6.4)$ & $3.5 \pm 2.1(1.1-5.0)$ & $5.3 \pm 2.4(2.5-6.8)$ & $5.7 \pm 2.9(2.5-8.3)$ \\
\hline \multicolumn{5}{|l|}{ NRS of LBP } \\
\hline All participants $(\mathrm{n}=7)$ & $5.4 \pm 1.7(4-9)$ & $4.1 \pm 1.2(3-6)^{a)}$ & $3.3 \pm 1.3(1-5)^{\mathrm{a}, \mathrm{b}}$ & $3.1 \pm 1.3(1-5)^{a)}$ \\
\hline Good responders $(n=4)$ & $6.2 \pm 1.9(5-9)$ & $4.8 \pm 1.3(3-6)$ & $3.3 \pm 1.7(1-5)$ & $3.3 \pm 1.0(2-4)$ \\
\hline Limited responders $(\mathrm{n}=3)$ & $4.3 \pm 0.6(4-5)$ & $3.3 \pm 0.6(3-4)$ & $3.3 \pm 0.6(3-4)$ & $3.0 \pm 2.0(1-5)$ \\
\hline
\end{tabular}

Values are presented as mean \pm standard deviation (range).

ATMS, abdominal trunk muscle strength; NRS, 11-point numerical rating scale; LBP, low back pain.

${ }^{\mathrm{a})} \mathrm{p}<0.05$ (vs. the outcome at 0 week), ${ }^{\text {b) }} \mathrm{p}<0.05$ (vs. the outcome at 4 week).

Table 5. Outcomes of the muscle strength, and the conditions of LBP and the locomotive syndrome

\begin{tabular}{lccc}
\multicolumn{1}{c}{ Outcomes } & Before the exercise at 0 week & After the exercise at 12 weeks & p-value \\
\hline AMTS $(\mathrm{kPa})$ & $4.4 \pm 2.1$ & $7.8 \pm 3.7$ & $<0.05$ \\
NRS of LBP & $5.4 \pm 1.7$ & $3.1 \pm 1.3$ & $<0.05$ \\
RDQ score & $6.0 \pm 4.1$ & $3.9 \pm 3.4$ & $<0.05$ \\
Stand-up test score & $3.7 \pm 1.0$ & $4.7 \pm 0.8$ & $<0.05$ \\
Two-step test score & $1.23 \pm 0.09$ & $1.46 \pm 0.07$ & $<0.05$ \\
Score of the GLFS-25 & $15.0 \pm 11.0$ & $12.4 \pm 10.0$ & 0.13 \\
\hline
\end{tabular}

Values are presented as mean \pm standard deviation.

LBP, low back pain; ATMS, abdominal trunk muscle strength; NRS, 11-point numerical rating scale; RDQ, the RolandMorris Disability Questionnaire; GLFD-25, 25-Question Geriatric Locomotive Function Scale. 
Table 6. Comparison of improvement of outcomes between the good and limited responders

\begin{tabular}{lccc}
\multicolumn{1}{c}{ Outcomes } & Good responders $(\mathbf{n}=\mathbf{4})$ & Limited responders $(\mathbf{n}=\mathbf{3})$ & p-value \\
\hline ATMS $(\mathrm{kPa})$ & $5.1 \pm 1.8$ & $1.1 \pm 0.7$ & $<0.05$ \\
NRS of LBP & $3.0 \pm 1.4$ & $1.3 \pm 1.5$ & 0.20 \\
RDQ score & $3.0 \pm 4.1$ & $1.0 \pm 1.0$ & 0.45 \\
Stand-up test score & $1.5 \pm 0.6$ & $0.3 \pm 0.6$ & $<0.05$ \\
Two-step test score & $0.24 \pm 0.05$ & $0.23 \pm 0.02$ & 0.96 \\
Score of the GLFS-25 & $4.3 \pm 5.9$ & $0.3 \pm 2.1$ & 0.33 \\
\hline
\end{tabular}

Values are presented as mean \pm standard deviation.

ATMS, abdominal trunk muscle strength; NRS, 11-point numerical rating scale; LBP, low back pain; RDQ, the RolandMorris Disability Questionnaire; GLFD-25, 25-Question Geriatric Locomotive Function Scale.

\section{Comparisons between good and limited responders}

The mean improvement in the stand-up test score was significantly better in good responders than in limited responders. The mean improvements in the NRS score of LBP, RDQ score, and the GLFS-25 score were also better in good responders than in limited responders, but these differences were not significant (Table 6).

\section{DISCUSSION}

This trial combined a device-driven abdominal trunk muscle strengthening program and stretching exercises. We wanted to offer participants a practical exercise program and evaluate the program as a comprehensive exercise treatment protocol. Our results indicated that the 12week exercise program-which consisted of stretching and strengthening exercises combined with use of our innovative device-was safe and not associated with any adverse events. The program effectively improved LBP, physical function, and abdominal trunk muscle strength in elderly patients.

Lumbar stabilization exercises are designed to improve stability in the lumbar spine and protect the spinal joints from microtraumas and degenerative changes. These exercises have been applied to treatment of CLBP [6,21-23]. The core muscles resemble a muscular box, with the diaphragm as the roof, abdominal muscles forming the front and sides, paraspinal muscles in the back, and pelvic floor muscles on the bottom [22]. Diaphragm contraction stabilizes the spine by increasing intra-abdominal pressure [22]. Brown et al. [24] proposed a bracing exercise for lumbar stabilization and reported that the bracing technique produced better spinal stabilization results than the hollowing exercise, which activates the deep core muscles such as the transverse abdominis. The bracing exercise further provided protection against sudden trunk perturbations $[25,26]$. During daily activities, ideal spinal stabilization coordinates all deep and superficial core muscles [27]. The abdominal contraction used with the device is similar to that used during abdominal bracing, creating a coordinated contraction of the deep and superficial core muscles at the anterolateral aspect, roof, and floor of the "muscular box" [18]. Lumbar stabilization exercises include "bridging" exercises such as the plank, side-bridge, and pelvic tilt. Okubo et al. [28] demonstrated that the superficial and deep muscles in the trunk were coactivated, but the activation level of each muscle differed according to the exercise. Many elderly patients with CLBP cannot perform bridging exercises due to deteriorated physical function, including pain and/or weakness in the trunk and extremities $[13,14]$. The device-driven abdominal trunk muscle strengthening exercises are performed while seated, did not stress lumbar spine movement. The exercise used in this trial did not induce pain in the trunk or extremities of elderly patients with CLBP. Because of this, participants continued to perform the exercises, eventually completing the entire program.

Several studies reported that trunk muscle strength was significantly lower in patients with LBP compared to asymptomatic participants [29-31]. A systematic review demonstrated that weak trunk muscle strength was associated with poor physical function, including impaired balance and increased incidence of falls in the elderly [32]. Granacher et al. [33] reported that, in elderly individuals with core instability, strength training improved trunk muscle strength, dynamic balance, and functional mobility. The device-driven exercise program in this 
trial-which included device-driven abdominal trunk muscle strengthening exercises-improved dynamic balance and functional mobility. These factors were using the locomotive syndrome risk test in elderly adults with CLBP.

The device-driven exercise program improved muscle strength, as measured using the device in all participants. Although most of the differences were not significant due to the small number of participants, good responders showed increased pain reduction and physical function compared to limited responders. In good responders, improvement of the stand-up test score was significantly better than that in limited responders. The stand-up movement requires adequate abdominal trunk muscle contraction and strength, as well as lower extremity muscle strength, adequate joint range of motion, flexibility, and balance [16]. Abdominal trunk muscle strengthening directly improved these important physical functions during activities of daily living. A lack of consensus on how to measure core muscle strength has weakened the impact of this research on determining optimal core stability exercises [27]. If we can easily and quickly measure core instability and muscle weakness, we can determine the outcomes and place proper emphasis on core muscle strengthening in patients. Our device could be a practical option for measuring core muscle strength. Further, it may also improve adherence to strengthening exercises. A systematic review reported that stretching produced the largest improvements in pain outcome measures. In contrast, strengthening exercises were most effective for improving functional outcomes among the various types of exercise therapies [11]. Device-driven strengthening exercises, combined with stretching, could help elderly participants improve their physical function and reduce LBP.

Previous studies reported that muscle strength measurement and device-driven strengthening exercises did not exert adverse effects in young adults $[17,18]$. Similarly, in the present study, use of the device for 12 weeks did not produce adverse effects in elderly patients with CLBP. These results demonstrate that both elderly and young patients can safely use the device. Of note, device usage increases intra-abdominal pressure. Hence, it is contraindicated in patients with histories of abdominal hernia surgery. It should also be used with great care in elderly patients with hypertension; cardiovascular, respiratory, cerebrovascular, or gynecological diseases; or other medical conditions.

The present study has several limitations, including the small sample size and lack of a control group. Improvements in CLBP and physical functions identified in the trial included the effect of stretching. A prospective, comparative controlled study a larger cohort is required to affirm these findings. Further studies are needed to compare outcomes among the device-driven exercises, other lumbar stabilization exercises, or other exercises prescribed for CLBP treatment. The study participants did not experience any device-related adverse effects or limitations. Future studies with a larger cohort, a wider age range, and both sexes are required to recognize drawbacks that might influence device utility.

Despite these limitations, our results demonstrated the efficacy of device-driven strengthening exercises, in combination with stretching, for improving CLBP, physical function, and abdominal trunk muscle strength in elderly patients with CLBP. The device-driven strengthening exercise was safe and could be a good treatment option to reduce CLBP and improve physical function, especially among elderly patients.

\section{CONFLICT OF INTEREST}

Nippon Sigmax Co. Ltd. provided the exercise device used in this clinical trial. The authors declare that they have no conflict of interest.

\section{AUTHOR CONTRIBUTION}

Conceptualization: Kato S. Data collection: Kato S, Kurokawa Y, Takahashi N, Yokogawa N, Yonezawa N, Shimizu T, Kitagawa R. Interpretation of data: Kato S, Demura S, Shinmura K. Formal analysis: Kato S, Kurokawa Y. Writing - original draft: Kato S. Writing - review and editing: Demura S, Tsuchiya H. Supervision: Tsuchiya H. Approval of the final manuscript: all authors.

\section{REFERENCES}

1. Dagenais S, Caro J, Haldeman S. A systematic review of low back pain cost of illness studies in the United States and internationally. Spine J 2008;8:8-20.

2. Bressler HB, Keyes WJ, Rochon PA, Badley E. The 
prevalence of low back pain in the elderly: a systematic review of the literature. Spine (Phila Pa 1976) 1999;24:1813-9.

3. Deyo RA. Diagnostic evaluation of LBP: reaching a specific diagnosis is often impossible. Arch Intern Med 2002;162:1444-8.

4. Poitras S, Brosseau L. Evidence-informed management of chronic low back pain with transcutaneous electrical nerve stimulation, interferential current, electrical muscle stimulation, ultrasound, and thermotherapy. Spine J 2008;8:226-33.

5. Bronfort G, Haas M, Evans R, Kawchuk G, Dagenais S. Evidence-informed management of chronic low back pain with spinal manipulation and mobilization. Spine J 2008;8:213-25.

6. Standaert CJ, Weinstein SM, Rumpeltes J. Evidenceinformed management of chronic low back pain with lumbar stabilization exercises. Spine J 2008;8:114-20.

7. Mayer J, Mooney V, Dagenais S. Evidence-informed management of chronic low back pain with lumbar extensor strengthening exercises. Spine J 2008;8:96113.

8. Slade SC, Keating JL. Trunk-strengthening exercises for chronic low back pain: a systematic review. J Manipulative Physiol Ther 2006;29:163-73.

9. Oesch P, Kool J, Hagen KB, Bachmann S. Effectiveness of exercise on work disability in patients with nonacute non-specific low back pain: systematic review and meta-analysis of randomised controlled trials. J Rehabil Med 2010;42:193-205.

10. Dagenais S, Tricco AC, Haldeman S. Synthesis of recommendations for the assessment and management of low back pain from recent clinical practice guidelines. Spine J 2010;10:514-29.

11. Hayden JA, van Tulder MW, Tomlinson G. Systematic review: strategies for using exercise therapy to improve outcomes in chronic low back pain. Ann Intern Med 2005;142:776-85.

12. Phillips EM, Schneider JC, Mercer GR. Motivating elders to initiate and maintain exercise. Arch Phys Med Rehabil 2004;85(7 Suppl 3):S52-7.

13. Rudy TE, Weiner DK, Lieber SJ, Slaboda J, Boston JR. The impact of chronic low back pain on older adults: a comparative study of patients and controls. Pain 2007;131:293-301.

14. Coyle PC, Velasco T, Sions JM, Hicks GE. Lumbar mo- bility and performance-based function: an investigation in older adults with and without chronic low back pain. Pain Med 2017;18:161-8.

15. Nakamura K. A "super-aged" society and the "locomotive syndrome". J Orthop Sci 2008;13:1-2.

16. Nakamura K, Ogata T. Locomotive syndrome: definition and management. Clin Rev Bone Miner Metab 2016;14:56-67.

17. Kato S, Murakami H, Inaki A, Mochizuki T, Demura $\mathrm{S}$, Nakase J, et al. Innovative exercise device for the abdominal trunk muscles: an early validation study. PLoS One 2017;12:e172934.

18. Kato S, Inaki A, Murakami H, Kurokawa Y, Mochizuki $\mathrm{T}$, et al. Reliability of the muscle strength measurement and effects of the strengthening by an innovative exercise device for the abdominal trunk muscles. J Back Musculoskelet Rehabil 2019 Oct 15 [Epub]. http://doi.org/10.3233/BMR-181419.

19. Kavcic N, Grenier S, McGill SM. Determining the stabilizing role of individual torso muscles during rehabilitation exercises. Spine (Phila Pa 1976) 2004;29:1254-65.

20. Ogata T, Muranaga S, Ishibashi H, Ohe T, Izumida $\mathrm{R}$, Yoshimura N, et al. Development of a screening program to assess motor function in the adult population: a cross-sectional observational study. J Orthop Sci 2015;20:888-95.

21. Richardson CA, Jull GA. Muscle control-pain control: what exercises would you prescribe? Man Ther 1995;1:2-10.

22. Akuthota V, Ferreiro A, Moore T, Fredericson M. Core stability exercise principles. Curr Sports Med Rep 2008;7:39-44.

23. Ferreira ML, Ferreira PH, Latimer J, Herbert RD, Hodges PW, Jennings MD, et al. Comparison of general exercise, motor control exercise and spinal manipulative therapy for chronic low back pain: a randomized trial. Pain 2007;131:31-7.

24. Brown SH, Vera-Garcia FJ, McGill SM. Effects of abdominal muscle coactivation on the externally preloaded trunk: variations in motor control and its effect on spine stability. Spine (Phila Pa 1976) 2006;31:E38793.

25. Grenier SG, McGill SM. Quantification of lumbar stability by using 2 different abdominal activation strategies. Arch Phys Med Rehabil 2007;88:54-62. 
26. Vera-Garcia FJ, Elvira JL, Brown SH, McGill SM. Effects of abdominal stabilization maneuvers on the control of spine motion and stability against sudden trunk perturbations. J Electromyogr Kinesiol 2007;17:556-67.

27. Akuthota V, Nadler SF. Core strengthening. Arch Phys Med Rehabil 2004;85(3 Suppl 1):S86-92.

28. Okubo Y, Kaneoka K, Imai A, Shiina I, Tatsumura M, Izumi S, et al. Electromyographic analysis of transversus abdominis and lumbar multifidus using wire electrodes during lumbar stabilization exercises. J Orthop Sports Phys Ther 2010;40:743-50.

29. Helewa A, Goldsmith C, Smythe H, Gibson E. An evaluation of four different measures of abdominal muscle strength: patient, order and instrument variation. J Rheumatol 1990;17:965-9.

30. Lee JH, Ooi Y, Nakamura K. Measurement of muscle strength of the trunk and the lower extremities in subjects with history of low back pain. Spine (Phila Pa 1976) 1995;20:1994-6.

31. Nourbakhsh MR, Arab AM. Relationship between mechanical factors and incidence of low back pain. J Orthop Sports Phys Ther 2002;32:447-60.

32. Granacher U, Gollhofer A, Hortobagyi T, Kressig RW, Muehlbauer T. The importance of trunk muscle strength for balance, functional performance, and fall prevention in seniors: a systematic review. Sports Med 2013;43:627-41.

33. Granacher U, Lacroix A, Muehlbauer T, Roettger K, Gollhofer A. Effects of core instability strength training on trunk muscle strength, spinal mobility, dynamic balance and functional mobility in older adults. Gerontology 2013;59:105-13. 